\title{
Enhancement of cisplatin sensitivity in lewis lung carcinoma by liposome-mediated delivery of a survivin mutant
}

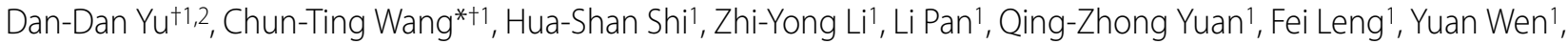 \\ Xiang Chen ${ }^{1}$ and Yu-Quan Wei ${ }^{1}$
}

\begin{abstract}
Background: A high concentration of cisplatin (CDDP) induces apoptosis in many tumor cell lines. CDDP has been administered by infusion to avoid severe toxicity. Recently, it has been reported that changes in survivin expression or function may lead to tumor sensitization to chemical and physical agents. The aim of this study was to determine whether a dominant-negative mouse survivin mutant could enhance the anti-tumor activity of CDDP.

Methods: A plasmid encoding the phosphorylation-defective dominant-negative mouse survivin threonine 34Talanine mutant (survivin T34A) complexed to a DOTAP-chol liposome (Lip-mS) was administered with or without CDDP in Lewis Lung Carcinoma (LLC) cells and in mice bearing LLC tumors, and the effects on apoptosis, tumor growth and angiogenesis were assessed. Data were analyzed using one-way analysis of variance(ANOVA), and a value of $P<$ 0.05 was considered to be statistically significant.

Results: LLC cells treated with a combination of Lip-mS and CDDP displayed increased apoptosis compared with those treated with Lip-mS or CDDP alone. In mice bearing LLC tumors and treated with intravenous injections of Lip-mS and/ or CDDP, combination treatment significantly reduced the mean tumor volume compared with either treatment alone. Moreover, the antitumor effect of Lip-mS combined with CDDP was greater than their anticipated additive effects.

Conclusion: These data suggest that the dominant-negative survivin mutant, survivin T34A, sensitized LLC cells to chemotherapy of CDDP. The synergistic antitumor activity of the combination treatment may in part result from an increase in the apoptosis of tumor cells, inhibition of tumor angiogenesis and induction of a tumor-protective immune response.
\end{abstract}

\section{Background}

There is a great deal of evidence that cisplatin (cis-diammine dichloroplatinum (II); CDDP) induces apoptosis in many tumor cell types. In the clinic, determining the greatest anti-tumoral efficiency using the lowest possible dose is a very difficult problem. Genetic therapy is considered to have enormous potential for resolving this issue.

A novel member of the inhibitor of apoptosis protein family (IAP), designated survivin [1], was recently identi-

*Correspondence: chtwang@163.com

1 State Key Laboratory of Biotherapy and Cancer Center, West China Hospital, West China Medical School, Sichuan University, Keyuan Road 4, Chengdu,

Sichuan, China

+ Contributed equally

Full list of author information is available at the end of the article fied by hybridization screening of human genomic libraries with the complementary DNA (cDNA) of a factor Xa receptor, effector cell protease receptor 1 [2]. Unlike all other IAPs, survivin is expressed during development and by common human cancers, but is undetectable or detected at extremely low levels in normal adult tissues[1]. Survivin therefore has become an attractive target for novel anticancer interventional agents[3]. In recent years, considerable effort has been expended towards counteracting survivin, including development of antisense oligonucleotides[4], hammerhead ribozymes[5], RNA interference[6,7], cancer vaccines[8] and dominant-negative mutants[9]. Several preclinical studies have already demonstrated that down-regulation of survivin expression or function could inhibit tumor 
growth, increase spontaneous and induced apoptosis and sensitize tumor cells to anticancer agents.

Phosphorylation of survivin at Thr 34 by the cyclindependent kinase cdc2 is believed to promote physical interaction between survivin and caspase-9, resulting in caspase-9 inhibition to reduce apoptosis[10]. It was reported that the survivin mutant Thr34TAla (survivin T34A) could abolish a phosphorylation site for cdc2cyclin B1 and prevent survivin binding to activated caspase-9[11]. This reduced tumor cell proliferative potential and led to caspase-dependent apoptosis in melanoma cell lines[9]. It also increased the apoptosis of tumor cells, inhibited tumor angiogenesis and induced a tumor-protective immune response [11]. It was found that greater efficiency was attained in suppression of murine breast cancer by using a plasmid encoding the phosphorylationdefective mouse survivin T34A mutant complexed to DOTAP-chol liposomes (Lip-mS) [11]. As a result, the present study was designed to determine whether Lip-mS could enhance the antitumor activity of CDDP chemotherapy and to explore the possible mechanisms of interaction between survivin targeting-agents and chemotherapy.

\section{Methods}

\section{Cell lines and culture conditions}

The Lewis Lung Carcinoma (LLC) cell line of C57BL/6 mouse origin was purchased from the American Type Culture Collection (ATCC, Rockville, MD), cultured in DMEM (Gibco BRL, Grand Island, N.Y.) supplemented with $10 \%$ heat-inactivated fetal bovine serum (FBS), and maintained in a humidified incubator at $37^{\circ} \mathrm{C}$ in a $5 \% \mathrm{CO}_{2}$ atmosphere.

\section{Plasmid DNA preparation}

The recombinant plasmid encoding the phosphorylationdefective mouse survivin threonine 34Talanine mutant (pORF9-msurvivinT34A, mS) and pORF9-mcs (null plasmid) were each purchased from InvivoGen Corporation (San Diego, CA, USA) and confirmed by restriction endonuclease analysis, PCR and DNA sequence analysis. The plasmid was prepared using the Endofree Plasmid Giga kit (Qiagen, Chatsworth, CA). Endotoxin levels of the prepared plasmid DNA were determined by Tachypleus Amebocyte Lysate (TAL). No genomic DNA, small DNA fragments, or RNA were detected in the plasmid DNA and the OD260/280 ratios of the DNA were between 1.8 and 2.0. The DNA was dissolved in sterile endotoxin-free water and stored at $-20^{\circ} \mathrm{C}$ until use.

\section{Preparation of DOTAP-chol liposome/plasmid DNA}

DOTAP was purchased from Avanti Polar Lipids (Alabaster, AL) and highly purified cholesterol (Chol) was purchased from Sigma (St. Louis, MO). DOTAP-chol liposomes were prepared using the procedure described previously[11]. DNA:liposome mixtures were also prepared in accordance with a previously-described method [12]. Briefly, DOTAP-chol (20 mM) and plasmid DNA stock solution diluted in $5 \%$ dextrose in water (D5W) were mixed in equal volumes to give a final concentration of $4 \mathrm{mM}$ DOTAP-chol, i.e., $150 \mu \mathrm{g}$ DNA in $300 \mu \mathrm{L}$ final volume (ratio, 1:2.6). These reagents were diluted and mixed at room temperature. The DNA solution was added to DOTAP-chol liposomes and rapidly mixed by pipetting up and down twice with the pipette tip. The DNA:liposome mixture thus prepared was precipitatefree and used for all the in vivo experiments. The size of the DNA fragments in the DNA:liposome mixture was determined to be in the range of 300-325 nm.

\section{Flow cytometric analysis}

LLC cells were seeded in a 6-well plate and incubated for $24 \mathrm{~h}$, then treated with normal saline (NS), CDDP, Lipnull, Lip-mS, or Lip-mS+CDDP (DNA at $1 \mu \mathrm{g} / \mathrm{mL}$ and CDDP at $4 \mu \mathrm{g} / \mathrm{mL}$ ). Forty-eight hours later, the cells were washed with PBS and resuspended in propidium iodide/ RNase A solution $(0.5 \mathrm{~mL})$, incubated at $37^{\circ} \mathrm{C}$ for $30 \mathrm{~min}$ and analyzed by flow cytometry.

\section{Animal studies}

Studies involving whole mice were approved by the Institute's Animal Care and Use Committee. Female C57BL/6 mice of 6 to 8 weeks old were purchased from the experimental animal center of Sichuan University (Chengdu, Sichuan Province, China) and challenged subcutaneously (s.c.) with LLC cells $\left(5 \times 10^{5}\right.$ cells in $50 \mu \mathrm{L}$ PBS $)$ in the right flank. Mice were randomly divided into 4 groups $(8$ mice per group) and treated with NS, Lip-mS, CDDP or Lip-mS + CDDP until the tumors had mean diameter of 3 $\mathrm{mm}$. Lip-mS was injected into mice via the tail vein at 5 $\mu \mathrm{g}$ per day once daily for 10 days (days 0 to 9 ) and CDDP (made in the Qilu Shandong Medical Factory) was injected into mice via the tail vein at $1 \mathrm{mg} / \mathrm{kg}$ per week (days 1, 8). Tumor size was determined by caliper measurement of the largest and perpendicular diameters every two days. Tumor volume was calculated according to the formula $\mathrm{V}=0.52 \mathrm{ab}^{2}$ ( $\mathrm{a}$ is the largest superficial diameter and $b$ is the smallest superficial diameter).

\section{Protein extraction and Western blot analysis}

Tumor tissue samples were ground into powder under liquid nitrogen by milling in mortar, and lysed in RIPA lysis buffer (50 mM Tris- $\mathrm{HCl}(\mathrm{pH} 7.4), 0.25 \%$ sodium deoxycholate, $150 \mathrm{mM} \mathrm{NaCl}, 1 \%$ nonidet P-40 (NP-40), 1 mM EDTA, $1 \mathrm{mM}$ NaF, $1 \mathrm{mM}$ Na3V4, $1 \mathrm{mM}$ phenylmethylsulfonyl fluoride). After being quantifided by Bradford assay, lysates were subjected to $12 \%$ SDS-PAGE (sodium dodecyl sulfate polyacrylamide gel) electropho- 
resis, electroblotted with Sartoblot onto a PVDF membrane (Millipore, Bedford, MA) for $1 \mathrm{hr}$ at $100 \mathrm{~V}$, and then membrane blots were blocked at $4^{\circ} \mathrm{C}$ in $5 \%$ non-fat dry milk, washed, and probed with rabbit anti-mouse Caspase 9 antibody (Abcam, Cambridge, United Kingdom) at 1:1000 and anti-actin antibody (Santa Cruz Biotechnology, Santa Cruz, CA) at 1:100. The blots were labeled with horseradish peroxidase-conjugated secondary antibody and visualized by chemiluminescence detection.

\section{Terminal deoxynucleotidyl transferase-mediated dUTP nick end-labeling (TUNEL) assay}

The mice were sacrificed by cervical dislocation on day 16 after the initiation of Lip-mS administration. Primary tumors were excised, fixed in $10 \%$ neutral-buffered formalin solution and embedded in paraffin. Contiguous 3-5 $\mu \mathrm{m}$ sections were mounted. In order to highlight the cells that were undergoing apoptosis, unstained sections mounted in silanized slides were subjected to fluorescent in situ TUNEL assay using an in situ apoptotic cell detection kit (Promega, Madison WI, USA), according to the manufacturer's protocol. Representative images were taken under a light microscope $(\times 200)$ in randomlyselected fields.

\section{CD31 immunohistochemical evaluation}

Immunohistochemistal analyses of microvessel formation were performed with goat anti-mouse CD31 antibody (Santa Cruz Biotechnology, Santa Cruz, CA) using the labeled streptavidin-biotin method. Briefly, sections were deparaffinized in xylol and rehydrated in a graded alcohol series. Antigen retrieval was carried out by autoclaving sections in retrieval buffer (10 mM EDTA citrate buffer, pH 6.0) for $3 \mathrm{~min}$ in saturated steam after up-pressure gaining (126, 1.6 bars, 23 psi). Endogenous peroxidase activity was blocked by incubation in 3\% hydrogen peroxide at room temperature in the dark for $20 \mathrm{~min}$. Non-specific binding of reagents was quenched by incubation of sections for $20 \mathrm{~min}$ in 5\% normal rabbit serum. Sections were then incubated with goat anti-mouse CD31 (dilution $1 / 200$ ) antibody overnight at $4^{\circ} \mathrm{C}$, followed by incubation with biotinylated rabbit antigoat IgG, and then streptavidin-biotin-horseradish peroxidase complex at $37^{\circ} \mathrm{C}$ for $1 \mathrm{~h}$. A negative control was included with each run by substituting the primary antibody with nonimmune rabbit serum. Cellular nuclei were counterstained with ameliorative Gill's hematoxylin. Representative images were taken under a light microscope $(\times 400)$ in randomly-selected fields.

\section{Statistical analysis}

Statistical analysis of the differences in tumor volume, percent apoptosis and microvessel density were performed using one-way analysis of variance(ANOVA). A value of $P<0.05$ was considered to be statistically significant.

\section{Results}

Enhancement of the anti-tumor effect of CDDP in vitro

In order to test the combined effect of Lip-mS with CDDP in vitro, we treated LLC cells with NS, CDDP (4 $\mu \mathrm{g} / \mathrm{mL}$ ), Lip-null (DNA at $1 \mu \mathrm{g} / \mathrm{mL}$ ), Lip-mS (DNA at 1 $\mu \mathrm{g} / \mathrm{mL})$ or Lip-mS + CDDP. Growth inhibition was analyzed by measuring cell viability with flow cytometric analysis to evaluate the effect of Lip-mS and CDDP on the induction of apoptosis in LLC cells. Lip-mS + CDDP treatment significantly increased the proportion (62.6\%) of sub-G1 cells (apoptotic cells) compared with the other treatments (NS, 8.7\%; CDDP, 8.3\%; Lip-null,9.0\%;Lip-mS, 44.6\%) (Fig. 1). Moreover, the interactive in vitro antitumor effect of the combined treatment was greater than the expected additive effect. The expected combined apoptotic effect was defined as follows: expected combined effect $=$ Lip-mS effect + CDDP effect - Lip-mS effect $\times$ CDDP effect. The Lip-mS and CDDP treatment can induce apoptosis $44.6 \%$ and $8.3 \%$ respectively, so the expected induction of apoptosis in the combined treatment should be $49.2 \%$. However, the actual induction of apoptosis in the combined treatment is $62.6 \%$, suggesting greater than additive treatment effect.

\section{Enhancement of the anti-tumor effects of CDDP in vivo}

The anti-tumor effect of Lip-mS in combination with CDDP was assessed in mice bearing LLC tumors. The tumor growth curves demonstrated that, relative to NS or CDDP alone, Lip-mS resulted in effective suppression of tumor growth, while the combined treatment had a supe-

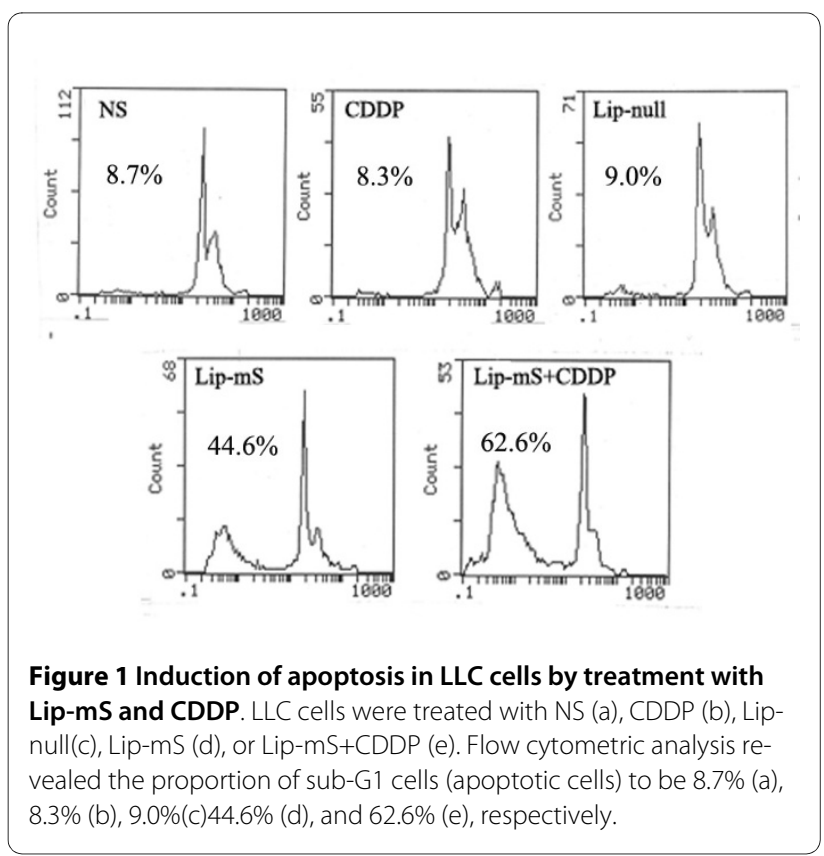


rior anti-tumor effect when compared with NS, Lip-mS or CDDP alone $(P<0.05)$ (Fig. 2$)$. Moreover, the interactive anti-tumor effects of the combined treatment were also greater than their expected additive effects. On day 16 after the initiation of Lip-mS administration, the tumor inhibitory rate (TIR) of the CDDP group was zero. the TIR of Lip-mS alone was $71.1 \%$ and the combination treatment group was $85.9 \%$. This suggests that combination treatment increased the inhibition, especially relative to CDDP $(P<0.05)$. In order to test by which possible mechanisms Lip-mS enhanced the anti-tumor effect of CDDP in vivo. The expression of caspase-9 in different treatment groups were detected by western blot. And tumor sections of each group were stained with TUNEL reagent and anti-CD31 antibody to evaluate the apoptotic rate and microvessel density. The details were described in Methods. Caspase-9 was found to be expressed to a higher extent in Lip-mS + CDDP treatment groups as compared to other groups(Fig. 3). And an apparent increase in the number of apoptotic cells was observed within the tumors treated with the combination of Lip$\mathrm{mS}$ and CDDP compared with other treatments $(P<0.05)$ (Fig. 4). Tumors of the NS and CDDP-treated groups exhibited high microvessel density, while the density was reduced in the Lip-mS-alone and combination treatment groups (Fig. 5). These data suggest that Lip-mS can cause increased apoptosis of tumor cells and inhibition of tumor angiogenesis, which may play important roles in

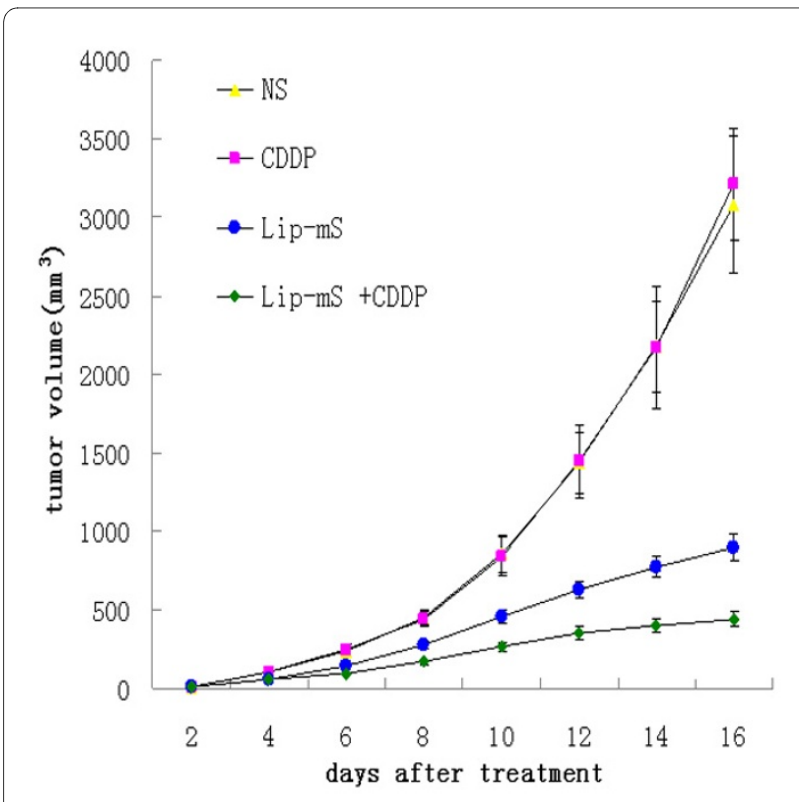

Figure 2 Lip-mS enhanced the antitumor effects of CDDP in vivo. Mice bearing LLC tumors were treated with NS, CDDP, Lip-mS or Lip$\mathrm{mS}+\mathrm{CDDP}$. Combination treatment reduced the mean tumor volume on day 16 when compared with the Lip-mS or CDDP treatment group $(P<0.05)$.

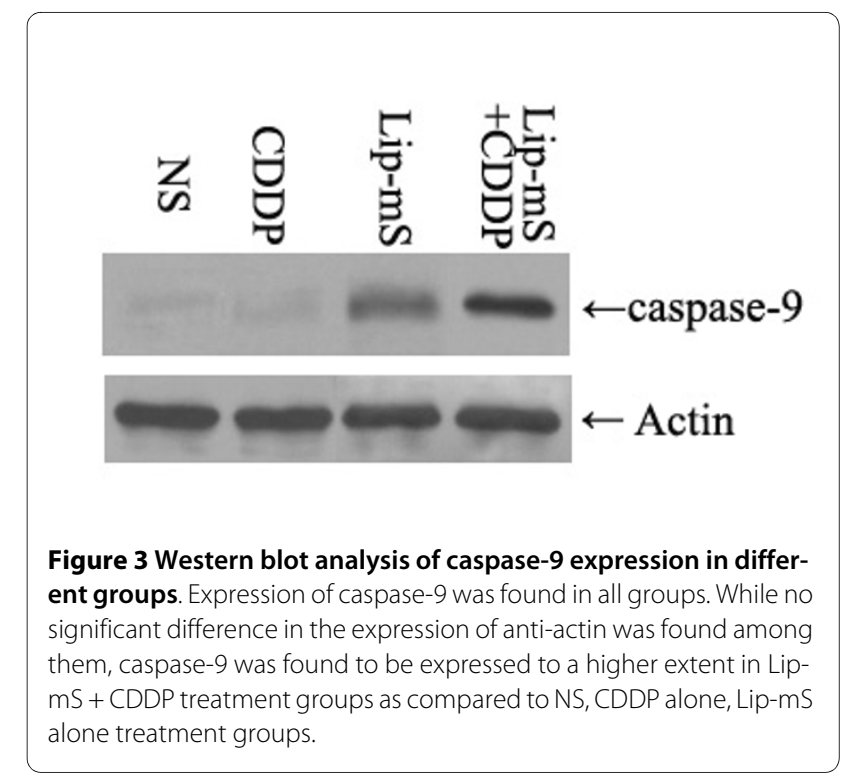

enhancement of the anti-tumor effects of chemotherapy in vivo.

\section{Discussion}

Survivin has received much greater attention in recent years, thanks not only to its anti-apoptotic effects, but also its relation to chemoresistance. It was reported that survivin acts constitutively in a panel of tumor cells, and approaches designed to inhibit survivin expression or function may lead to tumor sensitization to chemical and physical agents [13]. Hence, the combination of genetic and chemotherapeutic approaches has been a topic of great interest.

CDDP is widely used for the treatment of a variety of human tumors such as lung cancer[14]. CDDP is a wellknown DNA damaging agent, and it is currently thought that DNA platination is an essential first step in its cytotoxic activity[15]. However, continuous infusion or multiple administration of CDDP is an excellent regimen for cancer patients because of its adverse effects [16,17]. Therefore, approaches to improve the sensitivity to drug doses are a subject of intensive study in cancer care. Treatments combining genetic and chemotherapeutic approaches are a relatively new instrument in the fight against cancer.

Our study combining a Lip-mS genetic approach with CDDP significantly increased the anti-tumor effects of single chemotherapy. Moreover, the interactive antitumor effect of the combined treatment was greater than the expected additive effect. These data suggest that inhibition of survivin using a dominant-negative mutant, survivin T34A, can sensitize LLC cells to CDDP.

Reduction of apoptosis plays a very important role in tumor initiation, progression, and drug resistance. The major apoptosis-signaling pathways are the mitochon- 


\section{A}
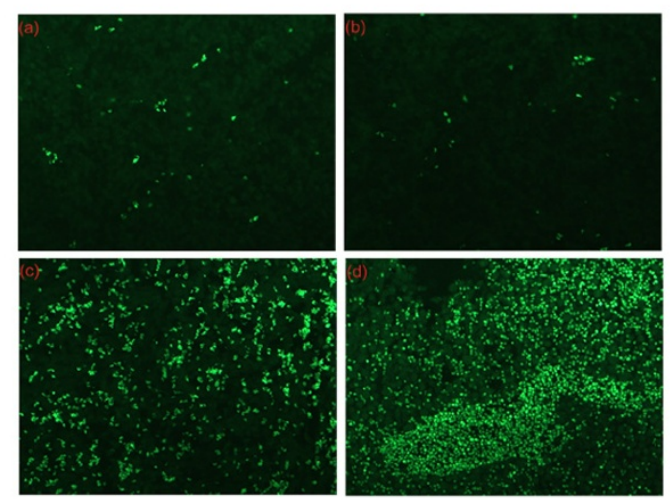

B

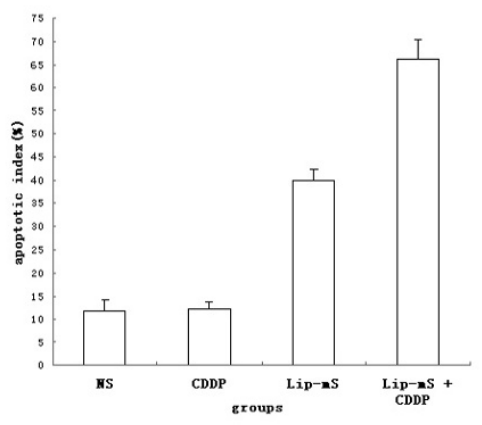

Figure 4 Combination of Lip-mS and CDDP enhanced the induction of apoptosis in vivo. Tissue sections from tumor-bearing mice treated with NS (a), CDDP (b), Lip-mS (c), or Lip-mS + CDDP (d) were stained with FITC-DUTP. Percent apoptosis was determined by counting the number of apoptotic cells and dividing by the total number of cells in the field (5 high power fields/slide). (A) Representative Field from each group. (B) Percent apoptosis in each group. Values were expressed as means \pm SE. An apparent increase in the number of apoptotic cells was observed within tumors treated with a combination of Lip-mS and CDDP compared with the other treatments $(P<0.05)$.

drial pathway and the death-receptor pathway. Several proteins that inhibit apoptosis have been identified, including the members of the bcl-2 family, such as bcl-2 and bcl-xL, and the IAPs. The anti-apoptotic proteins bcl-2 and bcl-xL block the apoptotic event of mitochondrial cytochrome $c$ release into the cytosol, and have been shown to mainly inhibit these two above-mentioned pathways. The gene encoding the IAP survivin has been cloned, and the protein characterized [18]. Survivin is thought to be expressed in the G2/M phase of the cell cycle in a cell cycle-regulated manner, and to be associated with microtubule formation of the mitotic spindle[19,20].

As a member of the IAP family, survivin can block apoptosis triggered by a variety of apoptotic-stimulating factors. It can directly bind to and inhibit caspase- 3 and caspase-7, which act at a common downstream part of the two major apoptotic pathways, and its overexpression

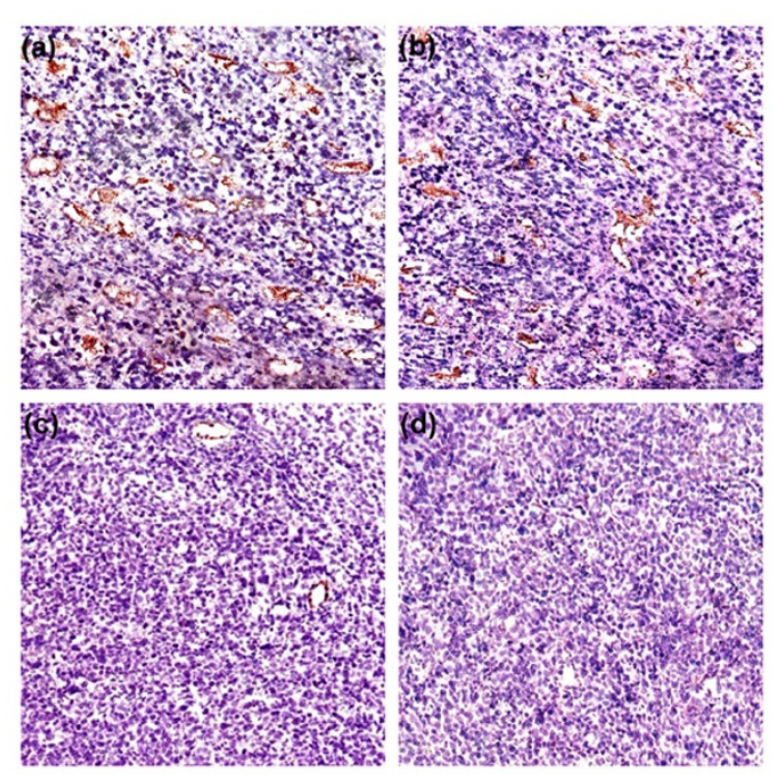

Figure 5 Inhibition of intra-tumoral angiogenesis assayed by CD31 staining of microvessels. Vascularization within tumors was detected by an antibody to CD31; representative images were taken under a light microscope $(\times 400)$ in randomly-selected fields. Tumors of the NS (a) and CDDP (b) treatment groups demonstrated high microvessel density, while those of the Lip-mS (c) and Lip-mS + CDDP (d) treatment groups showed apparent inhibition of angiogenesis.

in tumors has been implicated in resistance to a variety of apoptotic stimuli, including chemotherapy[17,20]. For this reason, the survivin antisense gene may facilitate both apoptotic pathways. Although survivin has long been considered a potential target for cancer therapy $[18,19,21-25]$, the use of antisense cDNA and oligonucleotides to inhibit its expression has only recently been described [26,27]. Previous studies have shown that reduction of survivin expression achieved by antisense strategies results in apoptotic cell death and sensitization to anticancer drugs in several tumor cell lines [26,27]. These results suggest that survivin expression is likely important for cell survival or resistance to chemotherapy in carcinomas.

CDDP acts in the G2/M phase of the cell cycle. Previous studies have shown that an increase in chemosensitivity is negatively correlated with survivin expression and positively correlated with rates of apoptosis[28]. The results of the study by Kojima et al are consistent with expression of survivin in the G2/M phase[29]. These observations are consistent with an earlier finding [26] that interaction between survivin and microtubules of the mitotic spindle apparatus is necessary to prevent a default induction of apoptosis at the G2/M phase of the cell cycle. And it is reported that cisplatin induced caspase- 9 activation and apoptosis in cisplatin-sensitive tumors[30]. Moreover, in a combination therapy experi- 
ment with CDDP, evidence was obtained that antisensemediated downregulation of survivin can sensitize tumor cells to chemotherapy in vitro and in vivo [29].

\section{Conclusions}

The survivin mutant had originally gained attention because it widely and specifically promoted apoptosis and enhanced chemotherapy, and its function and mechanism have been studied in various tumor types $[9,11,12,29]$. However, there are many aspects of its mechanisms that are still unclear. Our data suggest that the use of survivin T34A deserves further investigation as a useful approach to lung cancer therapy and in chemotherapy with CDDP. It was shown to down-regulate survivin expression and activity, to cause apoptosis in LLC cells, and to inhibit tumor growth. In addition, survivin T34A greatly enhances sensitivity to CDDP. These findings indicate the potential of this combination of a dominant-negative mutant--survivin T34A and administration of CDDP, or other chemotherapy, as a new therapeutic strategy for lung cancer.

\section{Competing interests}

The authors declare that they have no competing interests.

\section{Authors' contributions}

DDY carried out cell transfection, animal experiment, histologic analysis and drafted the manuscript. CTW participated in animal experiment, histologic analysis and helped to draft the manuscript. HSS and ZYL contributed to animal experiment. LP, FL, QZY and YW participated in plasmid DNA preparation. XC carried out Liposome preparation. YQW supervised experimental work and revised the manuscript. All authors read and approved the final manuscript.

\section{Acknowledgements}

This work is in part supported by the National 863 Project of China (2007AA021201).

\section{Author Details}

1State Key Laboratory of Biotherapy and Cancer Center, West China Hospital, West China Medical School, Sichuan University, Keyuan Road 4, Chengdu, Sichuan, China and '2Department of tumor Oncology, Henan People's Armed Police Corps Hospital, China

Received: 5 February 2010 Accepted: 12 May 2010

Published: 12 May 2010

\section{References}

1. Ambrosini G, Adida C, Altieri DC: A novel anti-apoptosis gene, survivin, expressed in cancer and lymphoma. Nat Med 1997, 3:917-921.

2. Altieri DC: Xa receptor EPR-1. FASEB J 1995, 9:860-865.

3. Sarela Al, Verbeke CS, Ramsdale J, Davies CL, Markham AF, Guillou PJ: Expression of survivin, a novel inhibitor of apoptosis and cell cycle regulatory protein, in pancreatic adenocarcinoma. Br J Cancer 2002, 86:886-892.

4. Sanwar JR, Shen WP, Kanwar RK, Berg RW, Krissansen GW: Effects of survivin antagonists on growth of established tumors and B7-1 immunogene therapy. J Natl Cancer Inst 2001, 93:1541-1552.

5. Pennati M, Colella G, Folini M, Citti L, Daidone MG, Zaffaroni N: Ribozymemediated attenuation of survivin expression sensitizes human melanoma cells to cisplatin-induced apoptosis. J Clin Invest 2002, 109:285-286.

6. Paduano F, Villa R, Pennati M, Folini M, Binda M, Daidone MG, Zaffaroni N Silencing of survivin gene by small interfering RNAs produces supraadditive growth suppression in combination with 17-allylamino-17- demethoxygeldanamycin in human prostate cancer cells. Mol Cancer Ther 2006, 5:179-186.

7. Jiang G, Li J, Zeng Z, Xian L: Lentivirus-mediated gene therapy by suppressing survivin in BALB/c nude mice bearing oral squamous cell carcinoma. Cancer Biol Ther 2006, 5:435-440.

8. Pisarev V, Yu B, Salup R, Sherman S, Gabrilovich DI: Full-length dominantnegative survivin for cancer immunotherapy. Clin Cancer Res 2003, 9:6523-6533.

9. Grossman D, Kim PJ, Schechner JS, Altieri DC: Inhibition of melanoma tumor growth in vivo by survivin targeting. Proc Nat/ Acad Sci USA 2001, 98:635-640.

10. Daniel S, O'Connor , Grossman Douglas: Regulation of apoptosis at cell division by p34cdc2 phosphorylation of surviving. Proc Natl Acad Sci USA 2000, 97:13103-13107.

11. McKay TR, Bell S, Tenev T, Stoll V, Lopes R, Lemoine NR, McNeish IA: Procaspase 3 expression in ovarian carcinoma cells increases survivin transcription which can be countered with a dominant-negative mutant, survivin T34A, a combination gene therapy strategy. Oncogene 2003, 22:3539-3547

12. Peng $X C$, Yang L, Wei $Y Q$, et al.: Efficient inhibition of murine breast cancer growth and metastasis by gene transferred mouse survivin Thr34TAla mutant. J Exp Clin Cancer Res 2008, 27:46.

13. Zaffaroni N, Daidone MG: Survivin expression and resistance to anticancer treatments: perspectives for new therapeutic interventions. Drug Resistance Updates 2002, 5:65-72.

14. Loehrer PJ, Einhorn LH: Drugs five years later. Cisplatin. Ann Intern Med 1984, 100:704-713.

15. Evans DL, Tilby M, Dive C: Differential sensitivity to the induction of apoptosis by cisplatin in proliferating and quiescent immature rat thymocytes is independent of the levels of drug accumulation and DNA adduct formation. Cancer Res 1994, 54:1596-1603.

16. Morazzoni F, Canevali C, Zucchetti M, Caroli S, Alimonti A, Petrucci F, Giudice G, Masoni E, Bedini AV: cis-Diamminedichloroplatinum(II) given in continuous infusion with concurrent radiotherapy to patients affected by inoperable lung carcinoma: a pharmacokinetic approach. Cancer Res Clin Oncol 1998, 24:137-43.

17. Kurihara N, Kubota T, Hoshiya Y, et al: Antitumor activity of cisdiamminedichloroplatinum (II) depends on its time $\times$ concentration product against human gastric cancer cell lines in vitro. J Surg Oncol 1995, 60:238-241.

18. Ambrosini G, Adida C, Altieri DC: A novel anti-apoptosis gene, survivin, expressed in cancer and lymphoma. Nat Med 1997, 3:917-921.

19. Li F, Ambrosini G, Chu EY, et al:: Control of apoptosis and mitotic spindle checkpoint by survivin. Nature 1998, 396:580-583.

20. Tamm I, Wang Y, Sausville E, et al.: IAP-family protein survivin inhibits caspase activity and apoptosis induced bf Fas(CD96), Bax, caspase, and anticancer drugs. Cancer Res 1998, 58:5315-5320.

21. Adida C, Crotty PL, MCGrath J, Berrebi D, Diebold J, Altieri DC: Developmentally regulated expression of the novel cancer antiapoptosis gene survivin in human and mouse differentiation. Am J Pathol 1998, 152:43-49.

22. Jaattela M: Escaping cell death: survival proteins in cancer. Exp Cell Res 1999, 248:30-47.

23. Kawasaki H, Altieri DC, Lu CD, Toyoda M, Tenjo T, Tanigawa N: Inhibition of apoptosis by survivin predicts shorter survival rates in colorectal cancer. Cancer Res 1998, 58:5071-5074.

24. LaCasse EC, Baird S, Korneluk RG, Mackenzie AE: The inhibitors of apoptosis IAPs) and their emerging role in cancer. Oncogene 1998, 17:3247-3259

25. Grossman D, MCNiff JM, Li F, Altieri DC: Expression of the apoptosis inhibitor, survivin, in nonmelanoma skin cancer and gene targeting in a keratinocyte cell line. Lab Invest 1999, 79:1121-1126.

26. Yamamoto T, Manome Y, Nakamura M, Tanigawa N: Down regulation of survivin expression by induction of the effector cell protease receptor1 reduces tumor growth potential and results in an increased sensitivity to anticancer agents in human colon cancer. Eur $\mathrm{J}$ Cancer 2002, 38:2316-2324

27. Olie RA, Simones-Wust AP, Baumann B, et al:: A novel antisense oligonucleotide targeting survivin expression induces apoptosis and sensitizes lung cancer cells to chemotherapy. Cancer Res 2000, 60:2805-2809 
28. Li F, Ackermann EJ, Bennett CF, et al:: Pleiotropic cell-division defects and apoptosis induced by interference with survivin function. Nat Cell Biol 1999, 1:461-466.

29. Hiromi K, Minoru I, et al:: Enhancement of Cisplatin Sensitivity in Squamous Cell Carcinoma of the Head and Neck Transfected With a Survivin Antisense Gene. Archoto head neck surg 2006, 132:682-685.

30. Kuwahara D: Caspase-9 regulates cisplatin-induced apoptosis in human head and neck squamous cell carcinoma cells. Cancer Letters 2000, 148:65-71.

doi: 10.1186/1756-9966-29-46

Cite this article as: Yu et al., Enhancement of cisplatin sensitivity in lewis

lung carcinoma by liposome-mediated delivery of a survivin mutant Journal of Experimental \& Clinical Cancer Research 2010, 29:46

Submit your next manuscript to BioMed Central and take full advantage of:

- Convenient online submission

- Thorough peer review

- No space constraints or color figure charges

- Immediate publication on acceptance

- Inclusion in PubMed, CAS, Scopus and Google Scholar

- Research which is freely available for redistribution

Submit your manuscript at www.biomedcentral.com/submit 